\title{
Enhanced neuroinflammation and pain hypersensitivity after peripheral nerve injury in rats expressing mutated superoxide dismutase 1
}

\author{
Julie V Berger ${ }^{1}$, Ronald Deumens², Stéphanie Goursaud', Sabrina Schäfer ${ }^{1}$, Patricia Lavand'homme³ \\ Elbert A Joosten ${ }^{2}$ and Emmanuel Hermans ${ }^{1 *}$
}

\begin{abstract}
Background: Neuroinflammation and nitroxidative stress are implicated in the pathophysiology of neuropathic pain. In view of both processes, microglial and astroglial activation in the spinal dorsal horn play a predominant role. The present study investigated the severity of neuropathic pain and the degree of glial activation in an inflammatory- and nitroxidative-prone animal model.
\end{abstract}

Methods: Transgenic rats expressing mutated superoxide dismutase 1 (hSOD1 ${ }^{\mathrm{G} 93 \mathrm{~A}}$ ) are classically used as a model for amyotrophic lateral sclerosis (ALS). Because of the associated inflammatory- and nitroxidative-prone properties, this model was used to study thermal and mechanical hypersensitivity following partial sciatic nerve ligation (PSNL). Next to pain hypersensitivity assessment, microglial and astroglial activation states were moreover characterized, as well as inflammatory marker gene expression and the glutamate clearance system.

Results: PSNL induced thermal and mechanical hypersensitivity in both wild-type (WT) and transgenic rats. However, the degree of thermal hypersensitivity was found to be exacerbated in transgenic rats while mechanical hypersensitivity was only slightly and not significantly increased. Microglial Iba1 expression was found to be increased in the ipsilateral dorsal horn of the lumbar spinal cord after PSNL but such Iba1 up-regulation was enhanced in transgenic rats as compared WT rats, both at 3 days and at 21 days after injury. Moreover, mRNA levels of Nox2, a key enzyme in microglial activation, but also of pro-inflammatory markers (IL-1 $\beta$ and TLR4) were not modified in WT ligated rats at 21 days after PSNL as compared to WT sham group while transgenic ligated rats showed up-regulated gene expression of these 3 targets. On the other hand, the PSNL-induced increase in GFAP immunoreactivity spreading that was evidenced in WT rats was unexpectedly found to be attenuated in transgenic ligated rats. Finally, GLT-1 gene expression and uptake activity were shown to be similar between WT sham and WT ligated rats at 21 days after injury, while both parameters were significantly increased in the ipsilateral dorsal region of the lumbar spinal cord of hSOD $1^{\mathrm{G} 93 \mathrm{~A}}$ rats.

Conclusions: Taken together, our findings show that exacerbated microglial activation and subsequent inflammatory and nitroxidative processes are associated with the severity of neuropathic pain symptoms.

\footnotetext{
* Correspondence: emmanuel.hermans@uclouvain.be

'Group of Neuropharmacology, Institute of Neuroscience, Université

catholique de Louvain, Brussels, Belgium

Full list of author information is available at the end of the article
} 


\section{Background}

Neuropathic pain is characterized by altered processing of nociceptive signals, mainly at the "first pain synapse" located at the level of the spinal dorsal horn. A prime role is commonly assigned to glial cells, surrounding these "first pain synapses" [1,2]. Particularly, microglia and astroglia have been shown to play pivotal roles in the onset and maintenance of pain hypersensitivity after peripheral nerve injury [3]. In healthy conditions, microglia ensure immunosurveillance of the central nervous system (CNS) [4] and astroglia, among others, regulate synaptic transmission through active clearance of ions and neurotransmitters [5]. Upon nerve injury however, glial cells become activated, as classically characterized by hypertrophied morphology, increased proliferation and up-regulation of specific markers, like ionized-calcium binding adaptor molecule 1 (Iba1) for microglia, or glial fibrillary acidic protein (GFAP) for astroglia $[6,7]$. Among many different glial activation states, some have been specifically related to neuropathic pain [8], characterized notably by up-regulation of pro-inflammatory proteins such as Toll-like receptor 4 (TLR4) [9] or NADPH oxidase 2 (Nox2) [10]. Moreover, the release of the pro-inflammatory cytokine interleukin $1 \beta$ (IL-1 $\beta$ ) was also found to contribute to pain hypersensitivity [11]. In addition, activation of astrocytes may interfere with the capacity of these cells to buffer the excitatory transmitter glutamate, through altered expression and/ or function of astroglial glutamate transporters, GLAST and GLT-1, thereby affecting the pain synapse [12-14].

Although glial activation, and more specifically subsequent neuroinflammation and nitroxidative stress are known to strongly contribute to neuropathic pain [15-17], it remains unknown whether such parameters may influence the severity of pain symptoms. We herein addressed this issue by studying nerve injury inducedhypersensitivity in a rat model characterized by inflammatory- and nitroxidative-prone properties.

Classically used as a model for amyotrophic lateral sclerosis (ALS), transgenic rats expressing the mutated form of the human superoxide dismutase 1 (hSOD $1^{\mathrm{G} 93 \mathrm{~A}}$ [18] showing a toxic gain of function [19]) are characterized by excitotoxic insults and increased nitroxidative stress $[20,21]$. The high vulnerability of motor neurons to these pathological processes explains their progressive loss in adulthood, leading to paralysis and death from respiratory failure. Previous studies have indicated that cultured glial cells derived from the hSOD $1^{\mathrm{G} 93 \mathrm{~A}}$ model are more inclined to get activated, and once activated, the release of neuroactive mediators is amplified [22-24]. We therefore hypothesized that performing a peripheral nerve injury in hSOD $1^{\mathrm{G} 93 \mathrm{~A}}$ rats early before the onset of any symptom, will cause exacerbated glial responses and subsequently, an increased neuroinflammatory and nitroxidative environment in the spinal cord, with putative influence on the severity of neuropathic pain symptoms. All experiments were completed within the pre-symptomatic stage of transgenic animals, as no symptom of the disease (such as motor deficit or weight loss) can be detected before 150 days (unpublished observation, Goursaud S.). Both wildtype (WT) and transgenic rats aged 90 days underwent partial sciatic nerve ligation (PSNL) to study thermal and mechanical hypersensitivity up to 21 days after surgery. In addition, biochemical studies were performed to characterize microglial and astroglial activation states as well as inflammatory processes and regulation in the glutamate clearance system in the dorsal spinal cord.

\section{Methods}

\section{General}

All experiments were strictly performed respecting the European Community Council directive of 24 November 1986 (86-609/ECC) and the decree of 20 October 1987 (87-848/EEC). Transgenic Sprague-Dawley rats expressing hSOD $1^{\text {G93A }}$ (multiple copies of the transgene, [25]) were kindly provided by Dr R. Pochet (Université Libre de Bruxelles, Belgium). Noteworthy and as commonly documented [26], the phenotype of the rats in our colony underwent a drift over multiple consecutive generations, leading to a postponed onset (150 days of age) and a slow disease progression. Animals were kept in controlled conditions (temperature, relative humidity, 12 h light/dark cycle) with constant access to food and water. To identify transgenic rats, tail biopsies from newborn rat pups (5-7 days old) were used, and the presence of the transgene was probed by PCR on genomic DNA extracts as previously described [27].

In this study, a total of 93 Sprague-Dawley female rats (females are characterized by a later ALS onset, [28]) were used, of which 46 were wild-type (WT) and 47 were transgenic (hSOD $1^{\mathrm{G} 93 \mathrm{~A}}$ positive, heterozygous). When the experiments started, all rats were aged 90 days and all experiments were completed within the pre-symptomatic stage.

First, the inflammatory-prone phenotype of the transgenic rats was investigated in our experimental paradigm. PSNL [29] or sham surgeries were performed on WT and transgenic rats that were sacrificed on postoperative day 3 or 21 to study microglial Iba1 gene expression. A second cohort of animals was used to monitor thermal and mechanical hypersensitivity for 21 days after surgery. After sacrifice, spinal tissues were analyzed for cellular and molecular changes related to a neuroactive environment (microglial and astroglial activation, expression of inflammatory markers) as well as for the study of glutamate transporters. All experiments were performed in a blinded manner, where the experimenters were unaware of experimental conditions. 


\section{Animal surgeries}

At 90 days of age, WT or hSOD $1^{\text {G93A }}$ female rats were anesthetized with sevoflurane (8\% for induction; $4 \%$ for maintenance; oxygen as carrier gas) and a PSNL was performed as previously described, with slight modifications [29]. Briefly, the right sciatic nerve was exposed at the mid-thigh level after isolation from the surrounding tissues. With a 6-0 suture, one third to one half of the nerve was tightly ligated at the site corresponding to the nerve trunk above its bifurcation in peroneal and tibial branches. Muscle and skin layers were finally closed with a 2-0 suture. Sham surgeries were performed by simply exposing the nerve before suturing the wound without performing the ligation.

\section{Behavioral testing}

During the 2 weeks preceding surgeries, all animals used for behavioral analyses were habituated to the testing procedures.

To examine thermal hyperalgesia (Hargreaves test), rats were placed in separate transparent boxes, for at least 15 min before starting measurements. A beam of radiant heat was applied to the mid plantar surface of rat's hind paws (Paw Thermal Stimulator, San Diego University, USA) and paw withdrawal latencies (PWL) were measured for the ipsilateral and the contralateral sides, with a cut-off fixed at $20 \mathrm{~s}$ to avoid tissue damage. For each time-point, the PWL of at least four testing trials, each separated by a 10 min interval, were averaged per rat and normalized by the rat's own average PWL before surgery (baseline).

To assess mechanical allodynia (von Frey hair filament tests), rats were placed in the same boxes, on a metal mesh floor for at least $15 \mathrm{~min}$ to let them habituate to the experimental environment. The previously described up-down method was used with minor modifications to determine the $50 \%$ paw withdrawal threshold (PWT, [30]). In total, 9 different von Frey filaments (Stoelting Co, Dale, USA) were selected, with bending force from $0.4 \mathrm{~g}$ to $26 \mathrm{~g}$. The filaments were applied perpendicularly to the mid plantar surface of hind paws until bending, for $8 \mathrm{~s}$. A response was considered as positive only if the rat showed paw withdrawal with clear signs of aversion against the stimulus (i.e. postural movement, licking of the paw, maintaining the paw in up-lift position).

One day before surgery, baseline values were obtained for each rat. Post-operative assessments of pain behavior were performed on day $1,2,3,5,7,10,14,17$ and 20 post-surgery.

\section{Fresh tissue dissection}

Rats were euthanized with $\mathrm{CO}_{2}$ and decapitated. The spinal cord was rapidly isolated by hydro-extrusion and transferred to cooled phosphate-buffered saline (PBS) solution to carefully remove meninges. The lumbar enlargement (L3-L6) was isolated and ipsilateral and contralateral sides were separated using the ventral fissure as a reference. Subsequently, these halves were divided into dorsal and ventral parts using the central canal as a reference. For measurement of glutamate transporter activity (synaptosomes, see below), fresh tissue samples were used immediately. For quantitative PCR (qPCR) experiments, tissues were frozen in $1 \mathrm{~mL}$ TriPure isolation reagent (Roche, Mannheim, Germany) before further use.

RNA extraction, reverse transcription and real-time qPCR After thawing of samples, the ipsilateral dorsal quadrant of the lumbar spinal cord was mechanically dissociated in TriPure isolation reagent, followed by RNA extraction according to the manufacturer's protocol. Reverse transcription was carried out with $1 \mu \mathrm{g}$ of RNA using the iScript cDNA synthesis kit (BioRad, Nazareth, Belgium), in a total volume of $20 \mu \mathrm{L}$. Real-time qPCR were finally performed to specifically amplify Iba1, glutamate transporters (GLAST and GLT-1), cytokines (IL-1 $\beta$ ), TLR4, Nox2 and GAPDH (see Table 1). Reactions were carried out in a total volume of $25 \mu \mathrm{L}$, containing $2 \mathrm{ng}$ of cDNA sample, $350 \mathrm{nM}$ of both specific forward and reverse primers and the IQ SYBR ${ }^{\circledR}$ Green supermix $1 \times$ (BioRad, $100 \mathrm{mM} \mathrm{KCl,} 40 \mathrm{mM}$ Tris- $\mathrm{HCl} \mathrm{pH} 8.4,0.4$ $\mathrm{mM}$ each dNTP, $50 \mathrm{U} / \mathrm{ml}$ of iTaq DNA polymerase, 6 $\mathrm{mM} \mathrm{MgCl}_{2}$, SYBR Green I, $20 \mathrm{nM}$ fluorescein, stabilizers). The protocol consisted of 45 amplification cycles, each characterized by $15 \mathrm{~s}$ of denaturation at $95^{\circ} \mathrm{C}, 45 \mathrm{~s}$ of hybridization at $60^{\circ} \mathrm{C}$ and $15 \mathrm{~s}$ of elongation at $79^{\circ} \mathrm{C}$. The measurements were performed using the iCycler IQ multicolor real-time PCR detection system, while the analysis of raw data was performed via the "post run data analysis" software provided by BioRad.

\begin{tabular}{|c|c|c|}
\hline Gene & Sequence & Amplicon (bp) \\
\hline$\overline{\text { GAPDH }}$ & $\begin{array}{l}\text { F: 5'- GTCTCCTGTGACTTCAACAG -3' } \\
\text { R: 5'- AGTTGTCATTGAGAGCAATGC -3' }\end{array}$ & 76 \\
\hline |ba1 & $\begin{array}{l}\text { F: 5'- CAGAATGATGCTGGGCAAG -3' } \\
\text { R: 5'- CCTCCAATTAGGGCAACTCA -3' }\end{array}$ & 127 \\
\hline GLAST & $\begin{array}{l}\text { F: 5'- GATCGGAAACATGAAGGAGC -3' } \\
\text { R: 5'- CAAGAAGAGGATGCCCAGAG -3' }\end{array}$ & 121 \\
\hline GLT-1 & $\begin{array}{l}\text { F: 5'- GGTCAATGTAGTGGGCGATT -3' } \\
\text { R: 5'- GGACTGCGTCTTGGTCATTT -3' }\end{array}$ & 124 \\
\hline Nox2 & $\begin{array}{l}\text { F: 5'- CCTTTCCTGCATCTGGGTCTCC - -3' } \\
\text { R: 5'- CGCCCTTGCCTCCATTCTC -3' }\end{array}$ & 164 \\
\hline $\mathbb{I L}-1 \beta$ & $\begin{array}{l}\text { F: 5'- GGAAGGCAGTGTCACTCATTGTG -3' } \\
\text { R: 5'- GGTCCTCATCCTGGAAGCTCC -3' }\end{array}$ & 84 \\
\hline TLR4 & $\begin{array}{l}\text { F: 5'- GATTGCTCAGACATGGCAGTTTC -3' } \\
\text { R: 5'- CACTCGAGGTAGGTGTTTCTGCTAA -3' }\end{array}$ & 135 \\
\hline
\end{tabular}


For quantification, a relative standard curve was generated for each targeted gene by using a cDNA template mix (combining all experimental samples) used at serial dilutions. After plotting the threshold values of amplifications versus the logarithm of the amount of cDNA added for the reaction, the relative amount of the targeted gene in each sample condition was calculated. Values were finally normalized against the relative expression of the housekeeping gene GAPDH.

\section{Measurement of $D-\left[^{3} \mathrm{H}\right]$-aspartate uptake activity in spinal cord synaptosomes}

After dissection, fresh tissue was immediately used to prepare synaptosomes, as previously described [31], with few modifications. The ipsilateral and contralateral dorsal part of the lumbar (L3-L6) spinal cord were homogenized in $5 \mathrm{~mL}$ of ice-cold $\left(4^{\circ} \mathrm{C}\right) 0.32 \mathrm{M}$ sucrose solution by repeating 10 up-and-down movements in a prechilled Teflon glass homogenizer. The homogenates were then centrifuged at $1000 \mathrm{~g}$ for $10 \mathrm{~min}$ at $4^{\circ} \mathrm{C}$, and only the supernatants, containing the synaptosomes, were collected and stored on ice, while the pellets were resuspended in $5 \mathrm{~mL}$ of fresh $0.32 \mathrm{M}$ sucrose solution at $4{ }^{\circ} \mathrm{C}$. After 10 up-and-down movements, the homogenates were again centrifugated at $1000 \mathrm{~g}$ for $10 \mathrm{~min}$ at $4^{\circ} \mathrm{C}$. Supernatants were pooled and finally centrifuged at $17500 \mathrm{~g}$ for $30 \mathrm{~min}$ at $4^{\circ} \mathrm{C}$. After discarding the supernatants, the pellets, containing the synaptosomes vesicles, were homogenized with 10 up-and-down strokes in 500 $\mu \mathrm{L}$ of a Krebs-ringer buffer $(120 \mathrm{mM} \mathrm{NaCl}, 4.8 \mathrm{mM}$ $\mathrm{KCl}, 1.3 \mathrm{mM} \mathrm{CaCl}_{2}, 1.2 \mathrm{mM} \mathrm{MgSO}, 1.2 \mathrm{mM} \mathrm{KH}_{2} \mathrm{PO}_{4}$, $25 \mathrm{mM} \mathrm{NaHCO}_{3}, 6 \mathrm{mM}$ glucose, $\mathrm{pH}$ 7.6) at $4^{\circ} \mathrm{C}$. Protein concentrations were determined for each sample with the Bradford method. To assess glutamate transporter activity, D- $\left[{ }^{3} \mathrm{H}\right]$-aspartate (specific activity of $11.3 \mathrm{Ci} /$ mmol, PerkinElmer NEN, Zaventem, Belgium) was used as substrate (as aspartate and glutamate have similar affinities for glutamate transporters), at a tracer concentration of $50 \mathrm{nM}$. In a total volume of $500 \mu \mathrm{L}, 40 \mu \mathrm{g}$ of protein (synaptosome preparation) were then incubated with the substrate for $10 \mathrm{~min}$ at $37^{\circ} \mathrm{C}$. To evaluate the GLT-1-dependent uptake, the specific inhibitor dihydrokainic acid (DHK, $100 \mu \mathrm{M}$, Tocris, Bristol, UK) was added to the reaction. The suspension was immediately filtered through a GF/B glass fiber filter adapted 96-well plate (UniFilter GF/B, PerkinElmer), and washed three times with the Krebs-Ringer buffer at $4^{\circ} \mathrm{C}$. Filters were then dried overnight at $37^{\circ} \mathrm{C}$. Microscint $20(45 \mu \mathrm{L}$, PerkinElmer) was then added to each well of the filter plate, and after $24 \mathrm{~h}$, plates were counted with a Topcount ${ }^{\circledR}$ Microplate scintillation and luminescence counter (PerkinElmer). Results are expressed as pmol of D$\left[{ }^{3} \mathrm{H}\right]$-aspartate transported per min and per mg of protein.

\section{Immunohistochemistry and quantification}

On day 21 post-surgery, rats were anesthetized using ketamine $(80 \mathrm{mg} / \mathrm{kg})$ and xylazine $(10 \mathrm{mg} / \mathrm{kg})$, and then sacrificed by transcardiac perfusion with saline solution $(\mathrm{NaCl}$ $0.9 \%$ ) at $37^{\circ} \mathrm{C}$, followed by perfusion with paraformaldehyde solution (4\% PFA in PBS buffer) at $44^{\circ} \mathrm{C}$. The spinal cord was isolated via hydro-extrusion and stored overnight in the PFA solution for a post-fixation at $4^{\circ} \mathrm{C}$. Cryopreservation was performed by incubation in a $15 \%$ sucrose solution (in PBS buffer) for $24 \mathrm{~h}$ at $4^{\circ} \mathrm{C}$, followed by incubation in a $30 \%$ sucrose solution for 3 consecutive days at $4{ }^{\circ} \mathrm{C}$. The lumbar region of each spinal cord (L3-L6) was frozen using dry ice. The samples were finally stored at $-80^{\circ} \mathrm{C}$ until cryosectioning. In total, 20 spinal cord samples were serially cut at $-30^{\circ} \mathrm{C}$ to obtain twelve series of transverse $30 \mu \mathrm{m}$ thick sections which were collected on ThermoScientific superfrost Plus glass slides (VWR International, Leuven, Belgium). Sections were stored at $-20^{\circ} \mathrm{C}$ until further histological staining. After thawing and drying, every $12^{\text {th }}$ section was stained for Iba1 or GFAP. For Iba1, sections were washed 3 times $(3 \times 10 \mathrm{~min})$ under gentle shaking, in Tris-buffered saline (TBS) ( $\mathrm{pH} 7.6)$, before incubation in a blocking solution (5\% normal donkey serum (NDS), $1 \%$ Triton X-100, in TBS pH 7.6) for $1 \mathrm{~h}$ at $4^{\circ} \mathrm{C}$. The primary antibody against Iba1 (rabbit anti-rat Iba1, 1:1000, Wako Pure Chemical Ltd, Osaka, Japan), was diluted in a working solution containing $1 \%$ NDS and $1 \%$ Triton X-100 (in TBS, pH 7.4), for an overnight incubation at $4^{\circ} \mathrm{C}$. The next day, after 3 washing steps $(3 \times 10 \mathrm{~min})$ with TBS, sections were incubated with secondary antibody (donkey anti-rabbit-Alexa488 antibody, 1:100, Invitrogen, Merelbeke, Belgium) diluted in the same working solution, for $1 \mathrm{~h}$ at $4^{\circ} \mathrm{C}$. After 3 washes in TBS $(3 \times 10$ $\mathrm{min}$ ), sections were embedded in $80 \%$ glycerol in PBS, and finally cover-slipped.

The protocol of GFAP staining was similar, except for the washing buffer (use of TBS-T: TBS containing $0.3 \%$ of Triton X-100), the absence of a blocking step, and the antibody incubation conditions (use of a TBS-T solution containing the primary antibody rabbit anti-rat GFAP 1:1000 from DAKO Diagnostics, Heverlee, Belgium, and the overnight incubation at room temperature). The secondary antibody (donkey anti-rabbitAlexa488 antibody, 1:100, Invitrogen) was diluted in a TBS-T solution for $1 \mathrm{~h}$ at room temperature. Sections were finally embedded and cover-slipped.

Photo-micrographs of the ipsilateral and contralateral dorsal horns were taken using a fluorescent microscope (Olympus Ax70, Paes, Zoeterwoude, The Netherlands) with a $4 \mathrm{x}$-objective and a grey-scale F-view cooled CCD camera. A total of 10 sections (L4-L5) per animal were analyzed using the cell profiler software CellP ${ }^{\circledR}$ (Olympus). The dorsal horn was delineated and after background subtraction, Iba1 and GFAP stained dorsal 
horns were analyzed. The percentage of dorsal horn showing positive immunostaining, also referred as "area fraction" (AF) was calculated for each photo-micrographs and data were finally plotted in a graph.

\section{Statistical analyses}

Data were expressed as means \pm standard error of the mean (SEM) and statistical analysis were performed with GraphPad Prism version 3.02 (GraphPad software, CA, USA). To compare the 4 different groups, a oneway ANOVA followed by the Tukey post-hoc test for multiple comparisons were used. A repeated measures ANOVA followed by a Tukey post-hoc test was used to compare the severity of pain hypersensitivity between WT and hSOD $1^{\text {G93A }}$ animals. Values of $p<0.05$ were considered as statistically significant. Symbols * were used to compare WT ligated $v s$ WT sham rats, symbols $\$$ were used to compare transgenic ligated $v s$ transgenic sham rats, and finally, symbols \# were used to compare WT ligated $v s$ transgenic ligated rats. Two symbols indicate a $p$ value $<0.01$ while 3 symbols represent a $p$ value $<0.001$.

\section{Results}

Up-regulation of Iba1 gene expression is enhanced in hSOD $1^{\text {G93A }}$ rats at 3 and at 21 days after PSNL

Quantitative RT-qPCR measurements of the microglial marker Iba1 were performed on spinal cord samples in order to evaluate the inflammatory response after PSNL (Figure 1). Ipsilateral lumbar dorsal quadrants were dissected from WT or transgenic rats, at 3 days and at 21 days after surgery. At the early post-operative timepoint (Figure 1A), WT ligated rats showed only a discrete increase in Iba1 mRNA levels as compared to their control group. In contrast, transgenic ligated rats showed a clear and statistically significant increase in gene expression of the microglial marker. The increase in Iba1 gene expression induced by PSNL was moreover significantly higher in transgenic rats as compared to WT animals. Importantly, mRNA expression of Iba1 did not differ between sham groups. At 21 days post surgery (Figure 1B), Iba1 gene expression appeared significantly increased in both ligated groups, but mRNA levels were clearly and significantly higher in samples from transgenic ligated rats when compared with WT ligated rats. Gene expression of the microglial marker was again found to be similar between both WT and transgenic sham groups.

\section{Rats expressing hSOD $1^{\mathrm{G} 93 \mathrm{~A}}$ show enhanced pain}

\section{hypersensitivity after PSNL}

Hypersensitivity to thermal and mechanical stimulation of hind paws was monitored during 21 days after sham or PSNL surgery, in both WT and transgenic rats

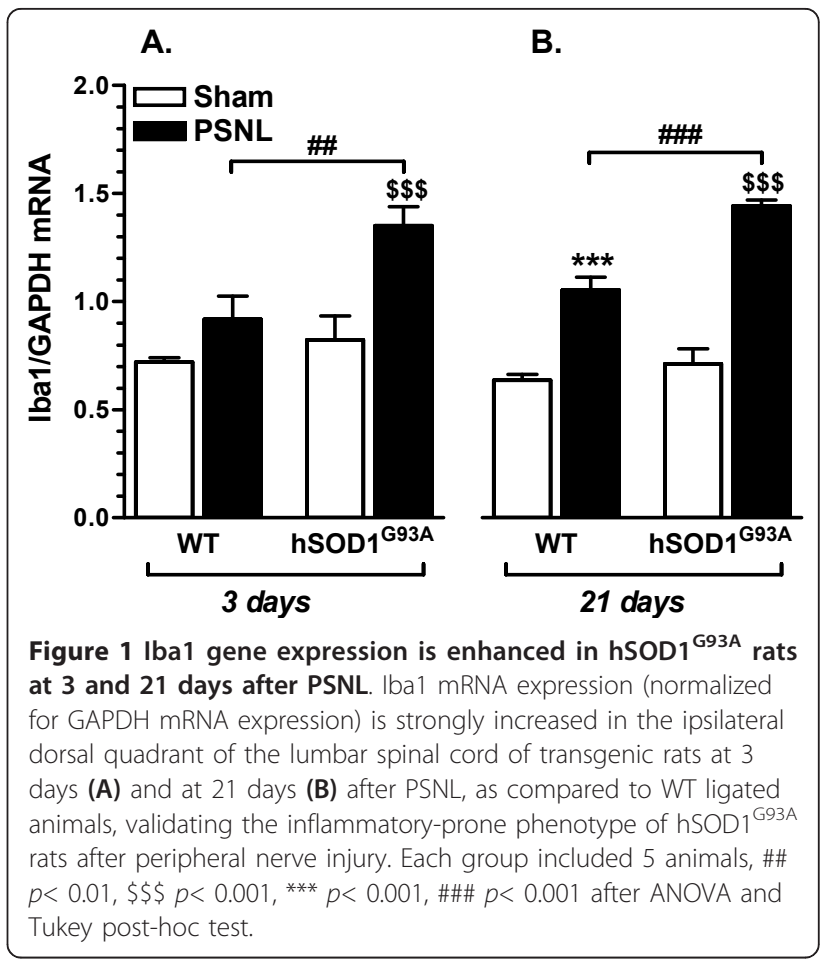

(Figure 2). Noteworthy, uninjured WT and hSOD1 ${ }^{\text {G93A }}$ rats showed similar baseline paw withdrawal latencies (PWL) and paw withdrawal thresholds (PWT). After PSNL however, a clear and significant decline in the withdrawal latency after thermal stimulation of ipsilateral hind paws was observed in ligated animals in comparison with their respective sham group (Figure 2A). Importantly, no difference in the PWL could be detected between both WT and transgenic sham groups throughout the period of thermal pain hypersensitivity assessment. The response was moreover unilateral as the withdrawal latency of contralateral paws was unaffected for both WT and hSOD ${ }^{\text {G93A }}$ rats (Figure $2 \mathrm{~B}$ ). Interestingly, thermal pain hypersensitivity after PSNL was more pronounced for transgenic compared to WT animals. Area under the curve analysis confirmed the significantly enhanced ipsilateral thermal pain hypersensitivity for transgenic ligated rats as compared to WT ligated rats (Figure 2E), while contralateral values were unchanged (Figure 2F).

Consistent with data from thermal stimulation, mechanical pain hypersensitivity testing indicated that PSNL caused a robust decrease in the ipsilateral 50\% PWT (Figure 2C) while no difference was observed on the contralateral side (Figure 2D). Both sham-operated groups showed similar 50\% PWT until the endpoint of mechanical pain hypersensitivity testing. However, the ipsilateral 50\% PWT did not differ between WT and transgenic rats after PSNL. Hence, the area under the 


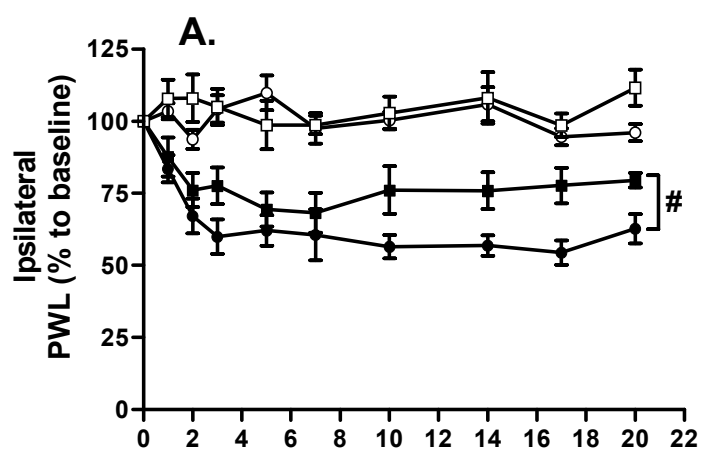

B.

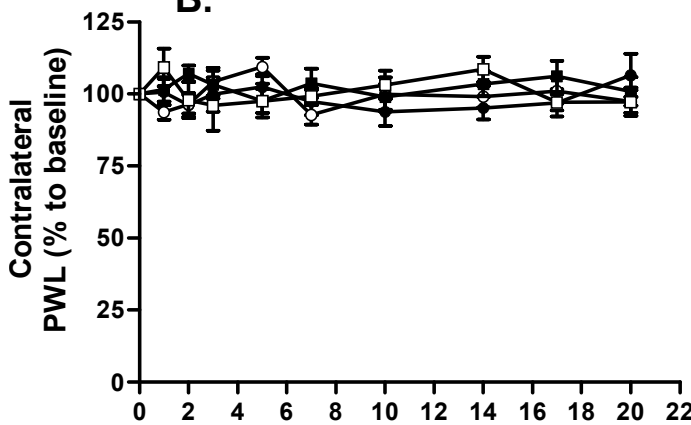

C.

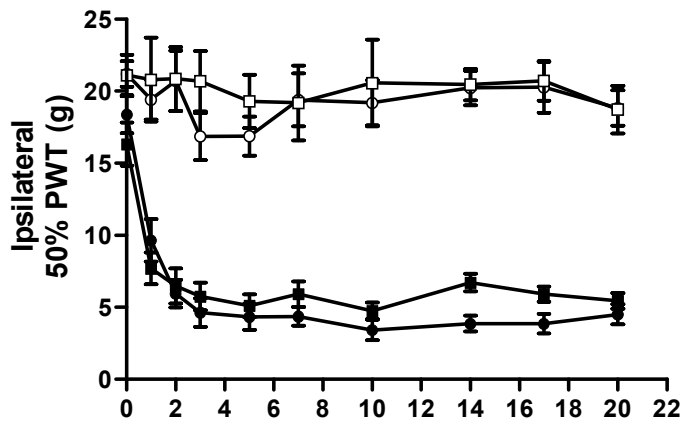

D.

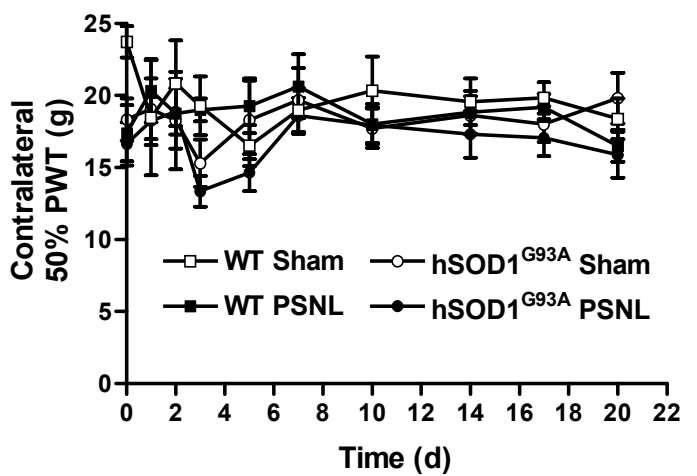

E.

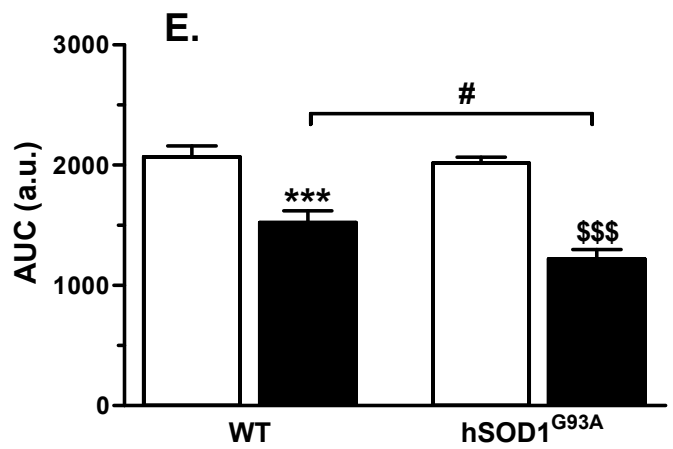

F.

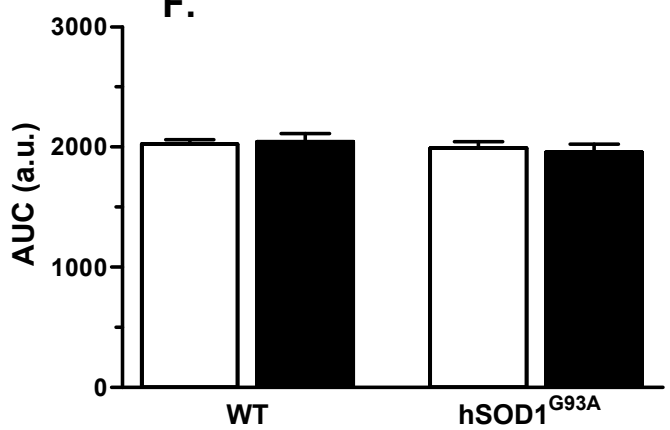

G.

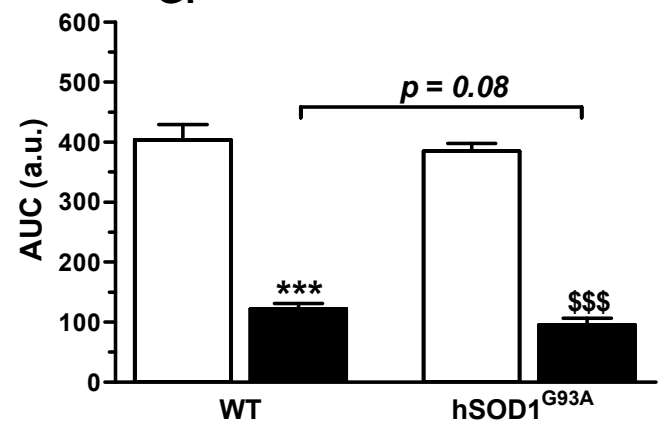

$\mathrm{H}$.

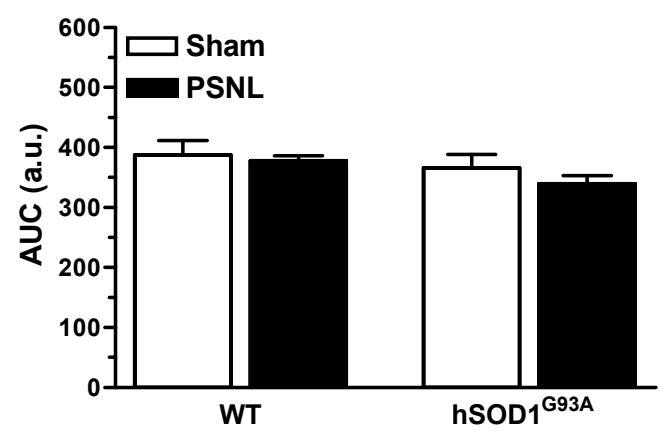

Figure 2 Rats expressing hSOD1 ${ }^{\text {G93A }}$ show enhanced pain hypersensitivity after PSNL. Rats were tested for thermal and mechanical hypersensitivity for 21 days after surgery. Paw withdrawal latencies (PWL) of the ipsilateral (A) or contralateral (B) hind paws after thermal stimulation, expressed as a percentage of the baseline PWL. PSNL induced a decrease of PWL in both WT and hSOD1 ${ }^{\mathrm{G} 93 \mathrm{~A}}$ rats on the ipsilateral side, compared to their respective sham controls (statistics not shown). The ANOVA repeated measures revealed enhanced pain hypersensitivity for hSOD ${ }^{\text {G93A }}$ rats compared to WT rats after PSNL. (C,D) 50\% paw withdrawal threshold (PWT) of the ipsilateral (C) or contralateral (D) hind paws after mechanical stimulation, expressed in grams. PSNL triggered mechanical hypersensitivity in both WT and transgenic rats (statistics not shown), but no statistical difference was evidenced between WT and transgenic rats after ligation. (E-H) Area under the curve (AUC) analysis of the corresponding graph confirmed the enhanced ipsilateral thermal hypersensitivity in transgenic rats compared to WT rats after PSNL (\#p < 0.05), while mechanical hypersensitivity only tends to be amplified in transgenic rats after lesion $(p=0.08)$; ANOVA and Tukey post-hoc test. Each group included 6 to 8 animals. 
curve analysis of the ipsilateral side (Figure 2G) indicated a trend $(p<0.08)$ towards a difference between WT ligated and hSOD1 ${ }^{\mathrm{G} 93 \mathrm{~A}}$ ligated animals. No changes were noticed in the area under the curve analysis for the contralateral side (Figure 2H).

\section{Rats expressing hSOD ${ }^{\mathrm{G} 93 \mathrm{~A}}$ show enhanced lba1 protein} up-regulation at 21 days after PSNL in the dorsal horn of the spinal cord

Immunohistochemical analysis of Iba1 protein expression was performed on lumbar spinal cord sections from rats sacrificed at 21 days after surgery. In both WT and hSOD ${ }^{\mathrm{G} 93 \mathrm{~A}}$ sham-operated groups, microglia were characterized as small and ramified cells (Figure 3A and 3B). Similar observations were made in the contralateral dorsal horn of WT rats with sham or PSNL surgery (data not shown). In contrast, a marked increase in Iba1 immunoreactivity was evidenced in the ipsilateral dorsal horn of both WT and hSOD $1^{\text {G93A }}$ ligated groups, where microglia were typically hypertrophied and less ramified (Figure 3C and 3D). To a lower extent, such hypertrophied microglia were also observed in the contralateral dorsal horn of the hSOD $1{ }^{\mathrm{G} 93 \mathrm{~A}}$ rats with PSNL (data not shown). Quantitative analyses showed that the ipsilateral Ibal area fraction was higher for rats with PSNL as compared to sham operated-rats. This difference was statistically significant for transgenic animals $(p<0.001)$ and showed a trend towards statistical significance for WT animals (Figure 3E). Overall, ipsilateral Iba1 protein expression associated with PSNL was found to be stronger in hSOD $1^{\mathrm{G} 93 \mathrm{~A}}$ rats as compared to WT animals $(p<0.05)$. Analysis of the contralateral dorsal horn demonstrated that PSNL significantly increased Iba1 area fraction in transgenic animals only (Figure 3F). Ipsilateral and contralateral Iba1 area fraction of the dorsal lumbar spinal horn were moreover found to be similar between both WT and transgenic sham groups. Finally, ipsilateral Iba1 area fraction values were interestingly found to be correlated $(p<0.001)$ with the percentage of change of the ipsilateral PWL from the baseline, on post-operative day 21 (Figure 3G). Noteworthy, as the PSNL damages both sensory and motor neuron processes in the sciatic nerve, increased Iba1 immunoreactivity was logically observed in the ipsilateral ventral horn of WT and transgenic ligated animals, though such responses were not further quantified.

\section{Rats expressing hSOD1 ${ }^{\text {G93A }}$ show impaired GFAP protein} up-regulation at 21 days after PSNL in the dorsal horn of the spinal cord

Immunodetection of GFAP expression was also performed on lumbar spinal cord sections in order to characterize the spreading of the astroglial response induced by PSNL in WT and hSOD $1^{\mathrm{G} 93 \mathrm{~A}}$ rats (Figure $4 \mathrm{~A}-\mathrm{D}$ ).
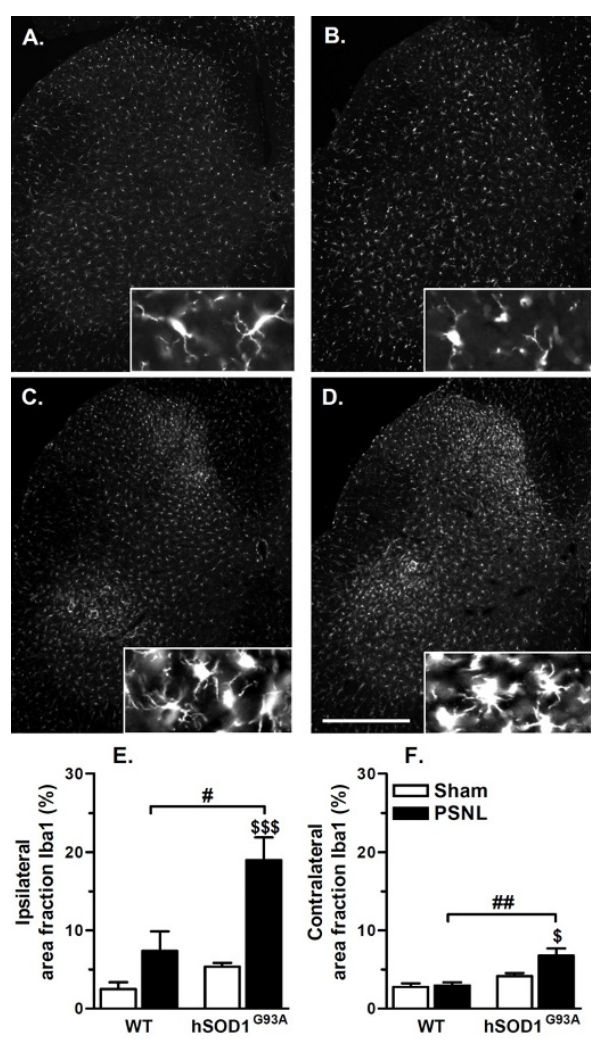

G.

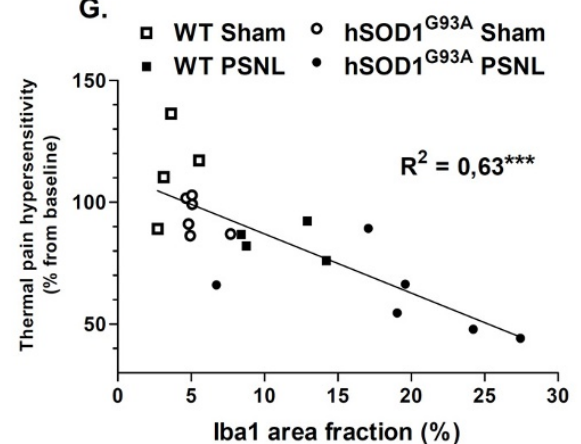

Figure 3 Increased lba 1 protein up-regulation in transgenic rats at $\mathbf{2 1}$ days after PSNL. (A-D) Immunohistological staining of the microglial marker Iba1 at 21 days after surgery, on the ipsilateral side of the lumbar spinal cord with a low and high magnification. $(\mathbf{A}, \mathbf{B})$ Microglia in both WT $(\mathbf{A})$ and transgenic (B) sham groups showed a ramified morphology. After PSNL, microglia exhibited a hypertrophied morphology, an effect which was found to be stronger in transgenic rats (D) as compared to WT rats (C) (scale bar represents $500 \mu \mathrm{m})$. (E,F) Quantification of the percentage of the total dorsal horn showing positive lba1 immunostaining. On the ipsilateral side (E), Iba1 protein up-regulation was found to be stronger in transgenic compared to WT rats after PSNL ( $\$ \$ p<$ 0.001; \# $p<0.05$ ). The contralateral side ( $\mathbf{F})$ showed a modest lba1 up-regulation only in transgenic rats at 21 days after PSNL ( $\$ p<$ 0.05 ; \#\# $p<0.01$ ). (G) The percentage of change in PWL from baseline on post-operative day 21 appeared to be correlated with the level of Iba1 up-regulation $\left(R^{2}=0.63\right.$ and $\left.p<0.001\right)$. WT groups included 4 animals whereas hSOD ${ }^{\mathrm{G} 93 \mathrm{~A}}$ groups included 6 animals; ANOVA and Tukey post-hoc test for statistical analyses. 

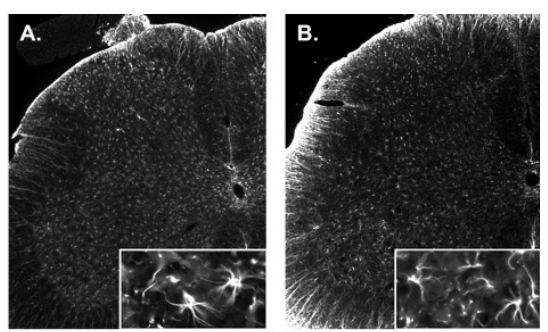
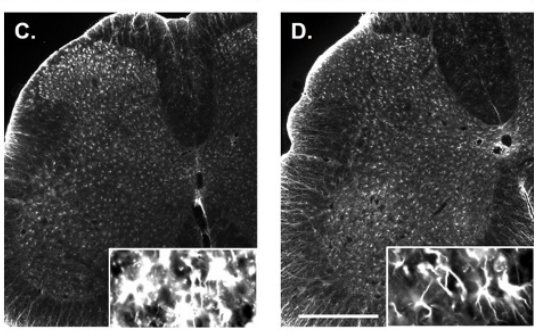

E.

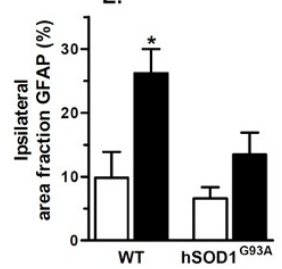

F.

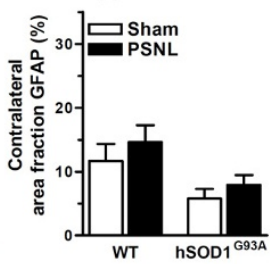

G.

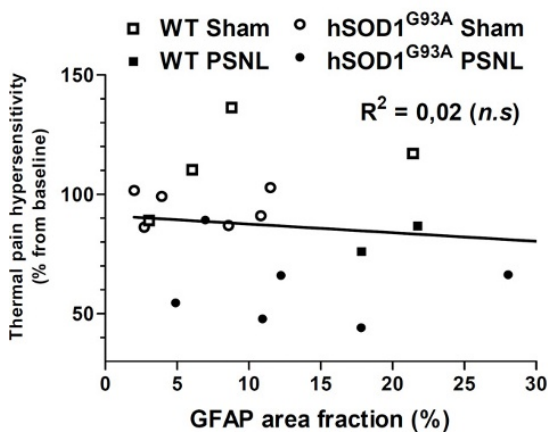

Figure 4 GFAP protein up-regulation is attenuated in hSOD ${ }^{\text {G93A }}$ rats at 21 days after PSNL. (A-D) Immunohistological staining of the astroglial marker GFAP at 21 days after surgery, on the ipsilateral side of the lumbar spinal cord with a low and high magnification. (A,B) Astrocytes in the ipsilateral dorsal horn of WT (A) or transgenic $(\mathbf{B})$ rats after sham operation showed a typical star-shaped morphology. At 21 days after PSNL, WT rats showed a strong astroglial response, characterized by cell hypertrophy and increased GFAP staining (C). In transgenic rats, this astroglial response was clearly attenuated (D) (scale bar represents $500 \mu \mathrm{m}$ ). $(\mathbf{E}, \mathbf{F})$ Quantification of the percentage of the total dorsal horn showing positive GFAP immunostaining. GFAP protein up-regulation was validated on the ipsilateral side (E) only for WT ligated rats, while transgenic rats showed a modest but non-significant increase in GFAP expression after PSNL. The contralateral side (F) did not show any statistical changes in GFAP area fraction. (G) The percentage of change in PWL from baseline on post-operative day 21 did not correlate with the level of GFAP up-regulation $\left(R^{2}=\right.$ 0.02). WT groups included 4 rats whereas hSOD $1^{\text {G93A }}$ groups included 6 rats; ANOVA and Tukey post-hoc test for statistical analyses.
Three weeks after lesion, GFAP immunoreactivity was found to be similar in the ipsilateral dorsal horn of both sham groups (Figure 4A and 4B), while the signal was clearly increased only in WT rats after ligation. With respect to cell morphology, immunoreactive cells appeared hypertrophied only in WT injured animals (Figure 4C), while few changes were observed in hSOD1 ${ }^{\text {G93A }}$ ligated rats (Figure 4D). Quantitative analysis of the area fraction of the ipsilateral dorsal horns from GFAP staining revealed significant increase following PSNL only for WT animals. For the transgenic model, GFAP area fraction was modestly but not significantly increased in comparison with sham littermates (Figure 4E). In the contralateral dorsal horn, no statistical changes could be detected for all animal groups (Figure 4F). Finally, no significant correlation was observed between ipsilateral GFAP area fraction and PWL on post-operative day 21 (Figure 4G). Here again, PSNL resulted in astroglial activation in the ipsilateral ventral horn of both WT and transgenic rats.

\section{Glutamate transporter expression and activity are increased after PSNL in hSOD $1^{\text {G93A }}$ rats}

Changes in glial glutamate handling likely contribute to altered pain transmission in the spinal cord of animals with peripheral nerve injuries. Therefore, the expression and activity of the two key glial glutamate transporters were compared in samples from WT and transgenic animals after PSNL. Quantitative PCR revealed that no significant changes in GLAST and GLT-1 mRNA levels were detected at 21 days after lesion in the ipsilateral dorsal quadrant of the lumbar spinal cord of WT rats. In contrast, a discrete but significant increase in GLAST expression was observed when comparing the groups of ligated animals (Figure 5A). Such difference was even more pronounced for GLT-1, as statistical analysis revealed increased expression associated with PSNL in transgenic rats when compared either to transgenic sham littermates or to WT ligated rats (Figure 5B).

Quantitative measures of aspartate uptake in synaptosomes prepared from lumbar dorsal spinal cord samples were performed in order to evaluate changes in the activity of glutamate transporters. Consistent with the increased gene expression of the transporters, increased uptake was measured in ipsilateral samples from hSOD $1^{\mathrm{G} 93 \mathrm{~A}}$ ligated rats whereas no change was observed for WT ligated animals (Figure 6A). In the tested conditions, GLT-1-dependent uptake (estimated using the specific blocker DHK) accounted for approximately $25 \%$ of total uptake. The use of this blocker evidenced that DHK-sensitive uptake was statistically increased only in samples from hSOD $1^{\mathrm{G} 93 \mathrm{~A}}$ injured rats but not in WT ligated animals (Figure 6C). For both WT and hSOD1 ${ }^{\text {G93A }}$ ligated groups, neither the total 


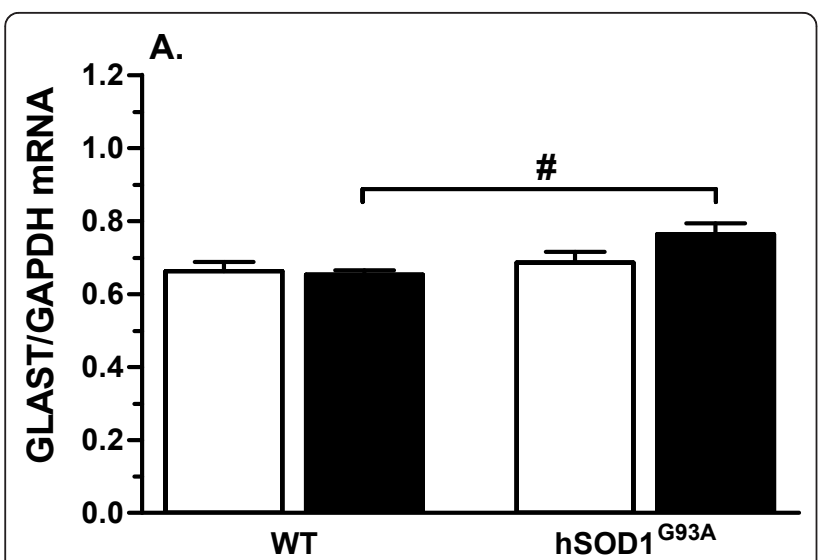

B.

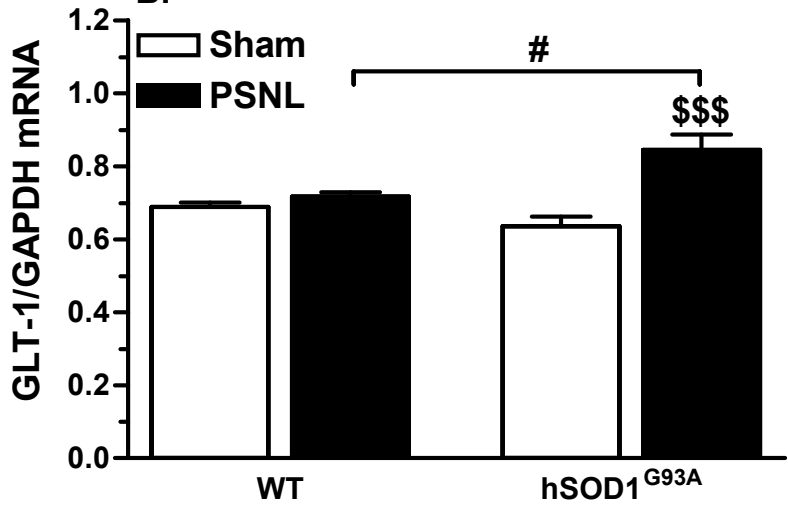

Figure 5 hSOD $1^{\text {G93A }}$ rats show higher mRNA levels of the glutamate transporters GLAST and GLT-1 after PSNL. (A) mRNA expression of the glutamate transporter GLAST (normalized for GAPDH mRNA expression) is slightly increased in the ipsilateral dorsal horn of transgenic rats when compared to WT animals at 21 days after PSNL (\#p<0.05). (B) mRNA levels of the glutamate transporter GLT-1 after GAPDH normalization are higher in the ipsilateral dorsal horn of transgenic rats at 21 days after PSNL when compared to the sham control rats or to WT ligated rats $(\$ \$ p<$ 0.001, \# $p<0.05$ ). Each group included 5 animals, ANOVA and Tukey post-hoc test for statistical analyses.

uptake nor the DHK-sensitive uptake were altered in samples from the contralateral dorsal horns (Figure 6B and D). Importantly, both gene expression and uptake capacities were found similar between WT and hSOD $1^{\text {G93A }}$ sham groups.

Increased gene expression of Nox2, IL-1 $\beta$ and TLR4 at 21 days after PSNL in hSOD $1^{\mathrm{G} 93 \mathrm{~A}}$ rats

To assess microglial activation, Nox 2 mRNA (catalytic subunit gp91 ${ }^{\text {phox }}$ ) [10] was quantified in the ipsilateral dorsal quadrant of the lumbar spinal cord at 21 days after surgery. While no difference could be detected between both sham groups, PSNL was shown to induce an increase in Nox2 gene expression. However, Nox2 mRNA up-regulation was found to be significant only for transgenic ligated rats when compared to their respective sham group, but also to WT ligated animals (Figure 7A). Because TLRs have previously been incriminated in neuropathic pain and TLR4 is the best characterized member of this family $[9,32]$, TLR4 gene expression was measured in the ipsilateral dorsal quadrant of the lumbar spinal cord at 21 days after surgery. At this time-point, TLR4 mRNA levels appeared strongly increased in transgenic ligated rats as compared to the other groups. In contrast, no significant PSNL induced-increase in this pro-inflammatory receptor was detected in WT rats (Figure 7B). Pro-inflammatory cytokines are moreover clearly incriminated in neuropathic pain [33] and more precisely, IL-1 $\beta$ was shown to be a key cytokine in the development and maintenance of pain hypersensitivity [11]. Hence, gene expression of IL$1 \beta$ was also quantified in the ipsilateral dorsal lumbar samples. Similarly, a substantial increase in IL-1 $\beta$ transcripts was only evidenced in transgenic animals after ligation when compared to the other groups (Figure $7 \mathrm{C}$ ).

\section{Discussion}

Microglial activation and subsequent neuroinflammatory/nitroxidative processes are known to directly influence neuropathic pain, however, such parameters were never studied after peripheral nerve injury in an animal model showing exacerbated inflammatory and nitroxidative properties, such as hSOD ${ }^{\text {G93A }}$ rats. First, we were able to verify that at the age chosen, uninjured transgenic rats did not differ from WT rats in baseline pain behavior. Second, no differences were observed between both WT and transgenic sham groups for all biochemical parameters tested in the dorsal region of the lumbar spinal cord. The present study demonstrates that in transgenic rats expressing hSOD $1^{\mathrm{G} 93 \mathrm{~A}}$, PSNL leads to: (1)- increased microglial Iba1 up-regulation at early (day 3 post surgery) and late (day 21 post surgery) timepoints; (2)- enhanced pain hypersensitivity; (3)- impaired astrogliosis at 21 days after lesion; (4)- increased expression and function of the glutamate transporters GLAST and GLT-1 at 21 days after injury; and (5)-increased gene expression of Nox2, TLR4 and IL-1 $\beta$ at 21 days after nerve injury.

Inflammatory reactions are exacerbated in the dorsal spinal cord of $\mathrm{hSOD} 1^{\mathrm{G} 93 \mathrm{~A}}$ rats after peripheral nerve injury

Over-expression of hSOD $1^{\text {G93A }}$ was already associated with inflammatory-prone properties in in vitro studies. Exposure of hSOD1 ${ }^{\text {G93A }}$-derived glial cells to an activating stimulus indeed leads to enhanced production of pro-inflammatory mediators and increased protein carbonylation, as compared to activated glia from control animals [22-24]. We here provide the first evidence that hSOD $1^{\text {G93A }}$ rats develop enhanced microglial activation 


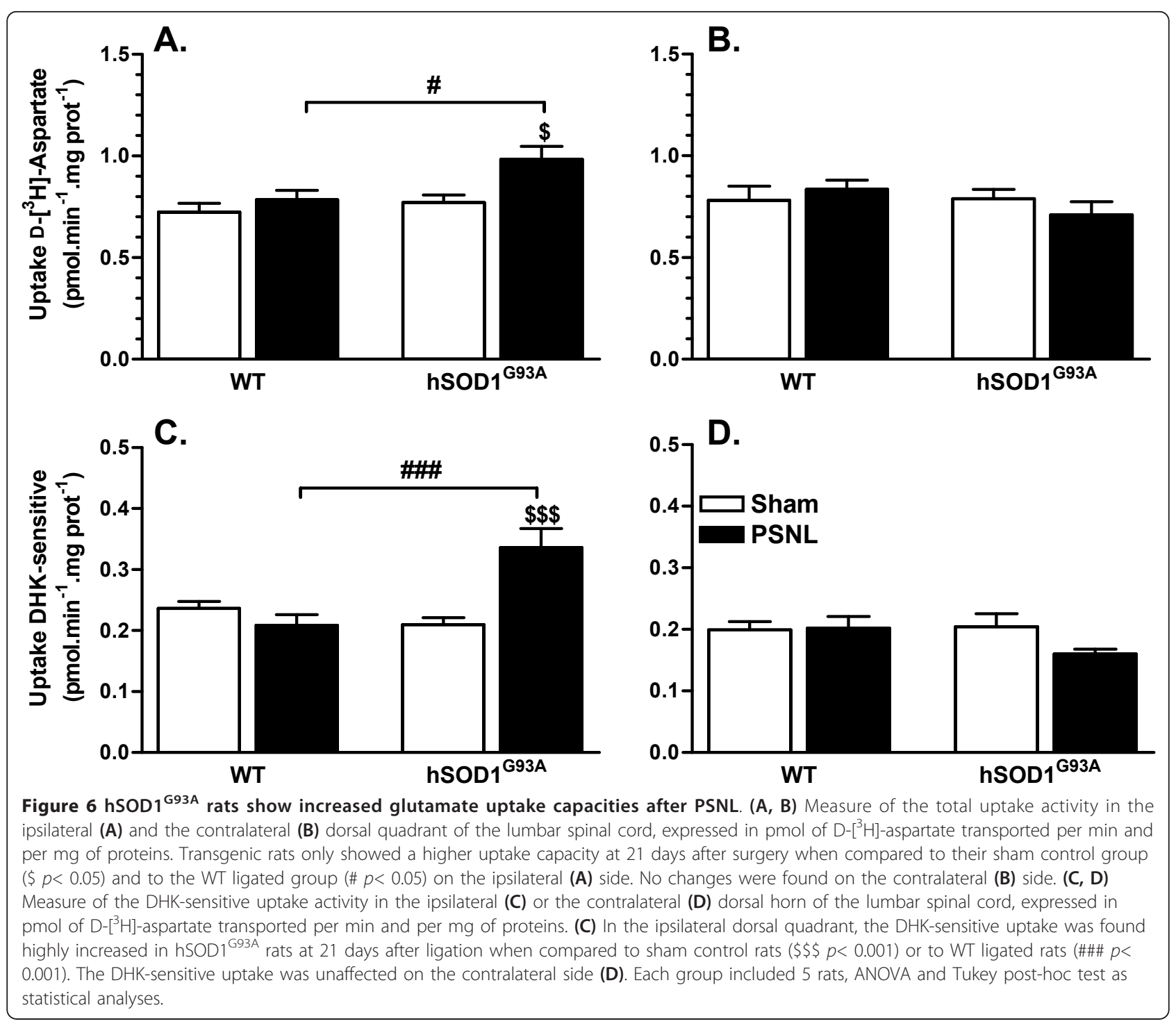

upon peripheral nerve injury, as evidenced by enhanced Iba1 gene up-regulation as early as 3 days after PSNL. Moreover, the neuropathy-induced increase in Iba1 gene and protein expression was also found to be exacerbated in transgenic rats at 21 days after PSNL, reinforcing the statement that microglial activation in hSOD $1^{\mathrm{G} 93 \mathrm{~A}}$ rats is clearly amplified upon injury.

The underlying mechanisms of exacerbated microglial responses may originate from the activating mutation of hSOD1, causing an imbalance in the redox equilibrium that ultimately promotes the expression and release of pro-inflammatory mediators, as well as reactive oxygen/ nitrogen species (ROS/RNS), after glial activation $[22,23,34]$. Importantly, similar Iba1 expression was measured for WT and hSOD $1^{\text {G93A }}$ sham animals on postoperative day 3 (mRNA level) and 21 (mRNA and protein levels), implicating that exacerbated activation is not observed in the absence of lesion. Furthermore, it also highlights that at this early pre-symptomatic stage of ALS, there is no glial activation and no molecular changes in the dorsal region of the lumbar spinal cord, as previously reported [35]. The effects obtained after PSNL appear therefore independent of the development of ALS.

\section{hSOD $1^{\text {G93A }}$ rats develop enhanced pain hypersensitivity} after PSNL

In comparison with WT rats, $\mathrm{hSOD} 1^{\mathrm{G} 93 \mathrm{~A}}$ animals showed enhanced thermal hypersensitivity following PSNL, while the increase in mechanical hypersensitivity was only mild and did not reach statistical significance. This discrepancy may be explained by a biased floor effect in the assessment of mechanical sensitivity, as nerve injury induced-PWTs are typically low in WT 


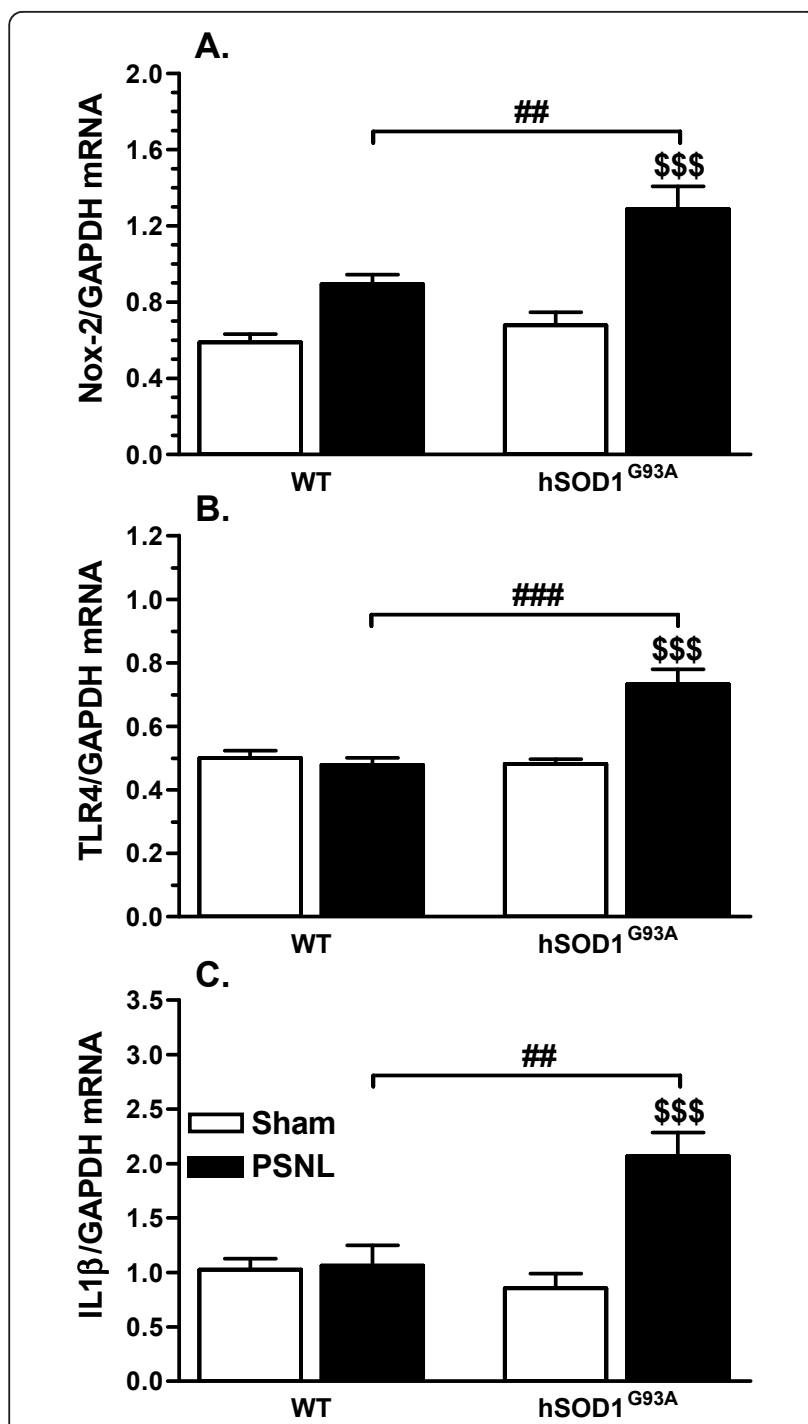

Figure $7 \mathrm{hSOD} 1^{\mathrm{G} 93 \mathrm{~A}}$ rats show increased gene expression of Nox2, TLR4 and IL-1 $\beta$ at 21 days after PSNL. (A) mRNA levels of Nox2 (normalized for GAPDH mRNA expression) are higher in the ipsilateral dorsal quadrant of the lumbar spinal cord of transgenic rats at 21 days after PSNL, when compared to the other groups ( $\$$ $\$ p<0.001$; \#\# $p<0.01$ ). (B) TLR4 gene expression was also increased in transgenic rats at 21 days after lesion when compared to their sham group ( $\$ \$ p<01$ ) or the WT ligated group (\#\# $p<$ 0.01). (C) IL-1 $\beta$ transcript level was significantly higher in transgenic ligated rats at 21 days after lesion when compared to sham littermates ( $\$ \$ p<0.001)$ or WT ligated rats (\#\# $p<0.01)$. Each group included 5 rats, ANOVA and Tukey post-hoc test as statistical analyses.

animals. Alternatively, a differential mechanism may underlie thermal as opposed to mechanical hypersensitivity. It is indeed proposed that mechanical hypersensitivity requires a specific population of unmyelinated fibers [36] while thermal hypersensitivity is dependent on the expression of a specific protein in nociceptors [37]. Thermal and mechanical hypersensitivities were moreover shown to be regulated by different pathways in an aquaporin-4 KO animal model [38] or after pharmacological treatment with ROS scavengers [10].

While microglial activation is known to contribute to the development of neuropathic pain [3,39], it has also been suggested to participate in the maintenance of this hypersensitive condition [40]. Hence, the increased PSNL induced-hypersensitivity in transgenic rats may reflect the activation state adopted by microglia in the dorsal horn of the spinal cord. Indeed, different activation states of microglia have been documented, suggesting that their response is specialized and dictated by the nature of the stimulus [41]. Only some of these phenotypic changes have been directly linked to neuropathic pain and have been qualified as "pain-related" [8]. Hence, it may be proposed that the activation state reached by microglia after PSNL differs between WT and transgenic rats, with distinct consequences on the severity of pain symptoms. Indeed, hSOD1 ${ }^{\text {G93A }}$ rats did not only show increased microglial activation, as evidenced with Iba1 gene and protein up-regulation, but also an increased gene expression of TLR4, which was absent in WT ligated rats. Because microglial TLR4 is linked to inflammation and pain [9], its high expression at late time-points supports the idea that $\mathrm{hSOD} 1^{\mathrm{G} 93 \mathrm{~A}}$ microglia adopt an exacerbated "pain-related" activation state after nerve injury. PSNL was moreover found to induce increased expression of the pro-inflammatory cytokine IL-1 $\beta$ in transgenic, but not in WT animals after 3 weeks. This cytokine was also clearly linked to neuropathic pain $[42,43]$ and may therefore participate in the enhanced pain hypersensitivity observed in transgenic rats. Finally, ROS production associated with Nox2 activity was previously shown to be critical for pain hypersensitivity, but also for the production of proinflammatory mediators [10]. Because transgenic rats show enhanced Nox2 gene expression after PSNL as compared to WT ligated rats, we conclude that the increased ROS production together with the exacerbated inflammatory reactions may contribute to the enhanced pain hypersensitivity. However, we cannot exclude the possibility that beside glial cells, inherent changes in dorsal horn sensory neurons due to hSOD $1^{\mathrm{G} 93 \mathrm{~A}}$ expression, are to some extent involved in the increased pain effects.

Surprisingly, we did not observe mirror-pain after PSNL. Although PSNL was originally associated with mirror-pain [29], works from different laboratories frequently led to inconsistent results [44-46]. Interestingly, we observed a modest microglial Iba1 up-regulation in the contralateral dorsal horn of transgenic rats. This observation supports the concept dissociating microglial responses and mirror-pain in the PSNL model [44]. 
hSOD1 ${ }^{\mathrm{G} 93 \mathrm{~A}}$ rats show impaired astroglial response in the spinal dorsal horn after PSNL

Consistent with previous investigations, PSNL induced an up-regulation of GFAP on the ipsilateral side of the spinal cord in WT rats $[47,48]$. However, such GFAP up-regulation appeared lower in hSOD $1^{\mathrm{G} 93 \mathrm{~A}}$ rats. Considering the increased lesion-associated hypersensitivity in these rats, this suggests that astrocytes do not exclusively exhibit pro-nociceptive effects in models of neuropathic pain. Indeed, it was already demonstrated that intrathecal grafting of PKC-activated astrocytes failed to induce pain behaviors [39]. Moreover, an inverse correlation has been reported between the intensity of mechanical hypersensitivity and GFAP immunoreactivity in the substantia gelatinosa at 3 months following complete sciatic nerve transection [49]. Hence, it cannot be excluded that the attenuated GFAP up-regulation in transgenic animals with PSNL may, to some extent, support the higher degree of neuropathic pain symptoms in these animals. The mechanisms involved in the attenuation of astroglial activation remain to be elucidated, but it is already known that the nature of cytokines is determinant in the resulting influence on astroglial reactivity [50]. Thus, it may be hypothesized that microglia, via the adopted activation state and the corresponding pattern of mediator expression, are able to differently drive astroglial responses. Of interest, IL- $1 \beta$, which was clearly increased in transgenic rats at 21 days post PSNL, has previously been shown to decrease GFAP expression in vitro [51,52].

\section{Glutamate clearance system is improved in the dorsal horn of hSOD $1^{\text {G93A }}$ animals after PSNL}

Glutamate transporter expression is influenced by a variety of factors, particularly inflammatory mediators $[13,14,53]$. Altered expression of glutamate transporters was already evidenced in several CNS pathologies [54-56], as well as in neuropathic pain models [57-59]. Biphasic regulation of these transporters has been reported after nerve injury with an up-regulation in the first days and a down-regulation at 2 weeks $[12,60,61]$. Glutamate transporter down-regulation was suggested to enhance pain hypersensitivity due to impaired glutamate handling. We found that GLAST and GLT-1 were both up-regulated at 21 days after PSNL in transgenic rats and, accordingly, uptake activity was found to be increased. Although surprising at first glance, these findings might be related to differential expression in astroglia and microglia, as it was previously reported after PSNL [62]. Indeed, while in astrocytes, GLAST and GLT-1 immunoreactivity was decreased, microglia on the other hand showed a de novo synthesis of both glutamate transporters, in accordance with other in vitro $[63,64]$ and in vivo [65] studies. The increased microglial expression of glutamate transporters may explain the overall increased uptake in transgenic ligated rats. Importantly, because transgenic rats showed increased pain hypersensitivity and microglial activation after PSNL, it suggests that microglia would not play a major role in regulating glutamate transmission.

\section{Conclusion}

Even though increased processing of experimental nociceptive stimuli was recently evidenced in ALS patient [66], the study of pain in the context of ALS was out of the scope of the present research which essentially took advantage of the characteristic of an animal model of the disease at a pre-symptomatic stage. Thus, we here demonstrated that rats expressing hSOD ${ }^{\mathrm{G} 93 \mathrm{~A}}$ develop more severe pain hypersensitivity after peripheral nerve injury. Although this finding may be explained by a variety of cellular and molecular mechanisms, our data suggest the involvement of specific microglial activation states such as those characterized by TLR4 and Nox 2 gene up-regulation, leading to an exacerbated inflammatory and nitroxidative environment. Moreover, sustained increases in IL- $1 \beta$ gene expression may also participate in the enhanced pain hypersensitivity. On the other hand, because astroglial activation was found to be attenuated in transgenic ligated rats, one may propose that activated astrocytes do not necessarily exert pro-nociceptive effects. Finally, the glutamate clearance system is affected in transgenic rats and this may additionally impact on the processing of pain signals in the spinal dorsal horn. The use of an animal model with SOD1 mutation showing more severe pain hypersensitivity and enhanced microglial activation after nerve injury may open new avenues in understanding neuropathic pain mechanisms.

\section{Acknowledgements}

We thank A. Lebbe, R. Lenaert, O. Ponchau and T. Timmerman for their excellent technical assistance. This study was supported by the National Fund for Scientific Research (FNRS, Belgium, contracts 3.4.560.07.F and 1. A198.08), by the DIANE research program of the Belgium's Walloon region (DGTRE) and by the Queen Elisabeth Medical Foundation (F.M.R.E.). JVB and SS are Research fellows of the F.N.R.S. and Télévie, respectively.

\section{Author details}

${ }^{1}$ Group of Neuropharmacology, Institute of Neuroscience, Université catholique de Louvain, Brussels, Belgium. 'Department of Anesthesiology, Maastricht University Medical Centre, Maastricht, The Netherlands. ${ }^{3}$ Department of Anesthesiology, Université catholique de Louvain, St. Luc Hospital UCL Medical School, Brussels, Belgium.

\section{Authors' contributions}

JVB was responsible for executing the entire research project, the statistical analyses and writing the manuscript. SG and SS assisted technically for rat breeding, aspartate uptake measurements and animal sacrifices. PL helped teaching the PSNL model. RD and EAJ supervised the immunohistochemical analyses and provided the material and facilities. EH directed the experiments and analyses and the writing of the manuscript. All authors read and approved the final manuscript. 


\section{Competing interests}

The authors declare that they have no competing interests.

Received: 18 January 2011 Accepted: 13 April 2011

Published: 13 April 2011

\section{References}

1. Ren $\mathrm{K}$, Dubner R: Interactions between the immune and nervous systems in pain. Nat Med 2010, 16:1267-1276.

2. Scholz J, Woolf CJ: The neuropathic pain triad: neurons, immune cells and glia. Nat Neurosci 2007, 10:1361-1368.

3. Tsuda M, Inoue K, Salter MW: Neuropathic pain and spinal microglia: a big problem from molecules in "small" glia. Trends Neurosci 2005, 28:101-107.

4. Nimmerjahn A, Kirchhoff F, Helmchen F: Resting microglial cells are highly dynamic surveillants of brain parenchyma in vivo. Science 2005 , 308:1314-1318.

5. Anderson CM, Swanson RA: Astrocyte glutamate transport: review of properties, regulation, and physiological functions. Glia 2000, 32:1-14.

6. Imai Y, Kohsaka S: Intracellular signaling in M-CSF-induced microglia activation: role of Iba1. Glia 2002, 40:164-174.

7. Pekny M, Nilsson M: Astrocyte activation and reactive gliosis. Glia 2005, 50:427-434.

8. McMahon SB, Malcangio M: Current challenges in glia-pain biology. Neuron 2009, 64:46-54.

9. Tanga FY, Nutile-McMenemy N, DeLeo JA: The CNS role of Toll-like receptor 4 in innate neuroimmunity and painful neuropathy. Proc Natl Acad Sci USA 2005, 102:5856-5861.

10. Kim D, You B, Jo EK, Han SK, Simon MI, Lee SJ: NADPH oxidase 2-derived reactive oxygen species in spinal cord microglia contribute to peripheral nerve injury-induced neuropathic pain. Proc Natl Acad Sci USA 2010, 107:14851-14856.

11. Kawasaki Y, Xu ZZ, Wang X, Park JY, Zhuang ZY, Tan PH, Gao YJ, Roy K, Corfas G, Lo EH, et al: Distinct roles of matrix metalloproteases in the early- and late-phase development of neuropathic pain. Nat Med 2008, 14:331-336.

12. Sung B, Lim G, Mao J: Altered expression and uptake activity of spinal glutamate transporters after nerve injury contribute to the pathogenesis of neuropathic pain in rats. J Neurosci 2003, 23:2899-2910.

13. Tilleux S, Hermans E: Down-regulation of astrocytic GLAST by microgliarelated inflammation is abrogated in dibutyryl cAMP-differentiated cultures. J Neurochem 2008, 105:2224-2236.

14. Tilleux S, Goursaud S, Hermans E: Selective up-regulation of GLT-1 in cultured astrocytes exposed to soluble mediators released by activated microglia. Neurochem Int 2009, 55:35-40.

15. Little JW, Doyle T, Salvemini D: Reactive nitroxidative species and nociceptive processing: determining the roles for nitric oxide, superoxide, and peroxynitrite in pain. Amino Acids 2010

16. Saab CY, Waxman SG, Hains BC: Alarm or curse? The pain of neuroinflammation. Brain Res Rev 2008, 58:226-235.

17. Watkins $L R$, Milligan ED, Maier SF: Glial activation: a driving force for pathological pain. Trends Neurosci 2001, 24:450-455.

18. Morrison BM, Morrison JH: Amyotrophic lateral sclerosis associated with mutations in superoxide dismutase: a putative mechanism of degeneration. Brain Res Brain Res Rev 1999, 29:121-135.

19. Okado-Matsumoto A, Fridovich I: Amyotrophic lateral sclerosis: a proposed mechanism. Proc Natl Acad Sci USA 2002, 99:9010-9014.

20. Barber SC, Shaw PJ: Oxidative stress in ALS: key role in motor neuron injury and therapeutic target. Free Radic Biol Med 2010, 48:629-641.

21. Van DP, Dewil M, Robberecht W, Van Den Bosch L: Excitotoxicity and amyotrophic lateral sclerosis. Neurodegener Dis 2005, 2:147-159.

22. Hensley K, Abdel-Moaty H, Hunter J, Mhatre M, Mou S, Nguyen K, Potapova T, Pye QN, Qi M, Rice H, et al: Primary glia expressing the G93ASOD1 mutation present a neuroinflammatory phenotype and provide a cellular system for studies of glial inflammation. I Neuroinflammation 2006, 3:2.

23. Liu Y, Hao W, Dawson A, Liu S, Fassbender K: Expression of amyotrophic lateral sclerosis-linked SOD1 mutant increases the neurotoxic potential of microglia via TLR2. J Biol Chem 2009, 284:3691-3699.

24. Weydt P, Yuen EC, Ransom BR, Moller T: Increased cytotoxic potential of microglia from ALS-transgenic mice. Glia 2004, 48:179-182.
25. Howland DS, Liu J, She Y, Goad B, Maragakis NJ, Kim B, Erickson J, Kulik J, DeVito $L$, Psaltis $G$, et al: Focal loss of the glutamate transporter EAAT2 in a transgenic rat model of SOD1 mutant-mediated amyotrophic lateral sclerosis (ALS). Proc Natl Acad Sci USA 2002, 99:1604-1609.

26. Herbik MA, Chrapusta SJ, Kowalczyk A, Grieb P: Maintenance of the rat transgenic model of familial amyotrophic lateral sclerosis expressing human SOD1G93A mutation. Folia Neuropathol 2006, 44:149-153.

27. Vermeiren C, Hemptinne I, Vanhoutte N, Tilleux S, Maloteaux JM, Hermans E: Loss of metabotropic glutamate receptor-mediated regulation of glutamate transport in chemically activated astrocytes in a rat model of amyotrophic lateral sclerosis. J Neurochem 2006, 96:719-731.

28. Suzuki M, Tork C, Shelley B, McHugh J, Wallace K, Klein SM, Lindstrom MJ, Svendsen $\mathrm{CN}$ : Sexual dimorphism in disease onset and progression of a rat model of ALS. Amyotroph Lateral Scler 2007, 8:20-25.

29. Seltzer Z, Dubner R, Shir Y: A novel behavioral model of neuropathic pain disorders produced in rats by partial sciatic nerve injury. Pain 1990, 43:205-218.

30. Chaplan SR, Bach FW, Pogrel JW, Chung JM, Yaksh TL: Quantitative assessment of tactile allodynia in the rat paw. J Neurosci Methods 1994, 53:55-63.

31. Bonnet JJ, Costentin J: Correlation between (3H)dopamine specific uptake and $(3 \mathrm{H}) \mathrm{GBR} 12783$ specific binding during the maturation of rat striatum. Life Sci 1989, 44:1759-1765.

32. Guo LH, Schluesener $H J$ : The innate immunity of the central nervous system in chronic pain: the role of Toll-like receptors. Cell Mol Life Sci 2007, 64:1128-1136.

33. Austin PJ, Moalem-Taylor G: The neuro-immune balance in neuropathic pain: involvement of inflammatory immune cells, immune-like glial cells and cytokines. J Neuroimmunol 2010, 229:26-50.

34. Henkel JS, Beers DR, Zhao W, Appel SH: Microglia in ALS: the good, the bad, and the resting. J Neuroimmune Pharmacol 2009, 4:389-398.

35. Graber DJ, Hickey WF, Harris BT: Progressive changes in microglia and macrophages in spinal cord and peripheral nerve in the transgenic rat model of amyotrophic lateral sclerosis. J Neuroinflammation 2010, 7:8.

36. Seal RP, Wang X, Guan Y, Raja SN, Woodbury CJ, Basbaum Al, Edwards RH: Injury-induced mechanical hypersensitivity requires C-low threshold mechanoreceptors. Nature 2009, 462:651-655.

37. Scherrer G, Low SA, Wang X, Zhang J, Yamanaka H, Urban R, Solorzano C, Harper B, Hnasko TS, Edwards RH, et al: VGLUT2 expression in primary afferent neurons is essential for normal acute pain and injury-induced heat hypersensitivity. Proc Natl Acad Sci USA 2010, 107:22296-22301.

38. Bao $F$, Chen $M$, Zhang $Y$, Zhao Z: Hypoalgesia in mice lacking aquaporin4 water channels. Brain Res Bull 2010, 83:298-303.

39. Narita M, Yoshida T, Nakajima M, Narita M, Miyatake M, Takagi T, Yajima Y, Suzuki T: Direct evidence for spinal cord microglia in the development of a neuropathic pain-like state in mice. J Neurochem 2006, 97:1337-1348.

40. Clark AK, Yip PK, Grist J, Gentry C, Staniland AA, Marchand F, Dehvari M, Wotherspoon G, Winter J, Ullah J, et al: Inhibition of spinal microglial cathepsin S for the reversal of neuropathic pain. Proc Natl Acad Sci USA 2007, 104:10655-10660.

41. Ransohoff RM, Perry VH: Microglial physiology: unique stimuli, specialized responses. Annu Rev Immunol 2009, 27:119-145.

42. Kawasaki Y, Zhang L, Cheng JK, Ji RR: Cytokine mechanisms of central sensitization: distinct and overlapping role of interleukin-1 beta, interleukin-6, and tumor necrosis factor-alpha in regulating synaptic and neuronal activity in the superficial spinal cord. J Neurosci 2008 , 28:5189-5194.

43. Reeve AJ, Patel S, Fox A, Walker K, Urban L: Intrathecally administered endotoxin or cytokines produce allodynia, hyperalgesia and changes in spinal cord neuronal responses to nociceptive stimuli in the rat. Eur $J$ Pain 2000, 4:247-257.

44. Clark AK, Gentry C, Bradbury EJ, McMahon SB, Malcangio M: Role of spinal microglia in rat models of peripheral nerve injury and inflammation. Eur J Pain 2007, 11:223-230.

45. Smits H, Ultenius C, Deumens R, Koopmans GC, Honig WM, van KM Linderoth B, Joosten EA: Effect of spinal cord stimulation in an animal model of neuropathic pain relates to degree of tactile "allodynia". Neuroscience 2006, 143:541-546.

46. Takaishi $\mathrm{K}$, Eisele JH Jr, Carstens E: Behavioral and electrophysiological assessment of hyperalgesia and changes in dorsal horn responses following partial sciatic nerve ligation in rats. Pain 1996, 66:297-306. 
47. Coyle DE: Partial peripheral nerve injury leads to activation of astroglia and microglia which parallels the development of allodynic behavior. Glia 1998, 23:75-83.

48. Ma W, Quirion R: Partial sciatic nerve ligation induces increase in the phosphorylation of extracellular signal-regulated kinase (ERK) and c-Jun $\mathrm{N}$-terminal kinase (JNK) in astrocytes in the lumbar spinal dorsal horn and the gracile nucleus. Pain 2002, 99:175-184.

49. Deumens R, Jaken RJ, Knaepen L, van dM I, Joosten EA: Inverse relation between intensity of GFAP expression in the substantia gelatinosa and degree of chronic mechanical allodynia. Neurosci Lett 2009, 452:101-105.

50. Reilly JF, Maher PA, Kumari VG: Regulation of astrocyte GFAP expression by TGF-beta1 and FGF-2. Glia 1998, 22:202-210.

51. Krohn K, Rozovsky I, Wals P, Teter B, Anderson CP, Finch CE: Glial fibrillary acidic protein transcription responses to transforming growth factorbeta 1 and interleukin-1 beta are mediated by a nuclear factor-1-like site in the near-upstream promoter. J Neurochem 1999, 72:1353-1361.

52. Oh YJ, Markelonis GJ, Oh TH: Effects of interleukin-1 beta and tumor necrosis factor-alpha on the expression of glial fibrillary acidic protein and transferrin in cultured astrocytes. Glia 1993, 8:77-86.

53. Okada K, Yamashita U, Tsuji S: Modulation of $\mathrm{Na}(+)$-dependent glutamate transporter of murine astrocytes by inflammatory mediators. J UOEH 2005, 27:161-170.

54. Beart PM, O'shea RD: Transporters for L-glutamate: an update on their molecular pharmacology and pathological involvement. Br J Pharmacol 2007, 150:5-17.

55. Sattler R, Rothstein JD: Regulation and dysregulation of glutamate transporters. Handb Exp Pharmacol 2006, 277-303.

56. Tilleux S, Hermans E: Neuroinflammation and regulation of glial glutamate uptake in neurological disorders. J Neurosci Res 2007, 85:2059-2070.

57. Binns BC, Huang Y, Goettl VM, Hackshaw KV, Stephens RL: Glutamate uptake is attenuated in spinal deep dorsal and ventral horn in the rat spinal nerve ligation model. Brain Res 2005, 1041:38-47.

58. Nie H, Weng HR: Impaired glial glutamate uptake induces extrasynaptic glutamate spillover in the spinal sensory synapses of neuropathic rats. J Neurophysiol 2010, 103:2570-2580.

59. Tawfik VL, Regan MR, Haenggeli C, Lacroix-Fralish ML, Nutile-McMenemy N, Perez N, Rothstein JD, DeLeo JA: Propentofylline-induced astrocyte modulation leads to alterations in glial glutamate promoter activation following spinal nerve transection. Neuroscience 2008, 152:1086-1092.

60. Cavaliere C, Cirillo G, Rosaria BM, Rossi F, De NV, Maione S, Papa M: Gliosis alters expression and uptake of spinal glial amino acid transporters in a mouse neuropathic pain model. Neuron Glia Biol 2007, 3:141-153.

61. Mirzaei V, Manaheji H, Maghsoudi N, Zaringhalam J: Comparison of changes in mRNA expression of spinal glutamate transporters following induction of two neuropathic pain models. Spinal Cord 2010.

62. Xin WJ, Weng HR, Dougherty PM: Plasticity in expression of the glutamate transporters GLT-1 and GLAST in spinal dorsal horn glial cells following partial sciatic nerve ligation. Mol Pain 2009, 5:15.

63. Nakajima K, Yamamoto S, Kohsaka S, Kurihara T: Neuronal stimulation leading to upregulation of glutamate transporter-1 (GLT-1) in rat microglia in vitro. Neurosci Lett 2008, 436:331-334.

64. Persson M, Brantefjord M, Hansson E, Ronnback L: Lipopolysaccharide increases microglial GLT-1 expression and glutamate uptake capacity in vitro by a mechanism dependent on TNF-alpha. Glia 2005, 51:111-120.

65. Lopez-Redondo F, Nakajima K, Honda S, Kohsaka S: Glutamate transporter GLT-1 is highly expressed in activated microglia following facial nerve axotomy. Brain Res Mol Brain Res 2000, 76:429-435.

66. Simone IL, Tortelli R, Samarelli V, D'Errico E, Sardaro M, Difruscolo O, Calabrese R, Francesco W, Livrea P, de TM: Laser evoked potentials in amyotrophic lateral sclerosis. J Neurol Sci 2010, 288:106-111.

doi:10.1186/1742-2094-8-33

Cite this article as: Berger et al.: Enhanced neuroinflammation and pain hypersensitivity after peripheral nerve injury in rats expressing mutated superoxide dismutase 1. Journal of Neuroinflammation 2011 8:33. 